\title{
Insight on passivity of high entropy alloys: thermal stability and ion transport mechanisms in the passive oxide film on $\mathrm{CoCrFeMnNi}$ surfaces
}

\author{
Luntao WANG ${ }^{1}$, Antoine SEYEUX ${ }^{1}$, Loïc PERRIERE ${ }^{2}$, Dimitri MERCIER ${ }^{1}$, Vincent MAURICE ${ }^{1}$, Philippe MARCUS $^{1 *}$ \\ ${ }^{1}$ PSL Research University, CNRS - Chimie ParisTech, Institut de Recherche de Chimie Paris, Physical Chemistry of Surfaces Group, 11 rue \\ Pierre et Marie Curie, 75005 Paris, France \\ ${ }^{2}$ Université Paris Est Creteil, CNRS, ICMPE, UMR7182, Thiais F-94320, France \\ Corresponding author: \\ E-mail address: philippe.marcus@chimieparistech.psl.eu (Philippe Marcus).
}

\begin{abstract}
The ion transport mechanisms in passive films pre-formed on CoCrFeMnNi high entropy alloy surfaces in acid solution were studied in-situ by time-of-flight secondary ion mass spectrometry upon oxidation in ${ }^{18} \mathrm{O}_{2}$ gas. The bilayer structure of the passive film, with outer $\mathrm{Fe}$, Co-rich and inner $\mathrm{Cr}$, Mn-rich layers, is thermally stable, but with reduction of Fe and Co oxides and formation of Mn oxide in the outer layer above $150^{\circ} \mathrm{C}$. At $170^{\circ} \mathrm{C}$, the transport mechanism is mainly outward cation diffusion with slower inward oxygen diffusion. The re-oxidation growth rate follows a parabolic law and the value of parabolic constant was determined.
\end{abstract}

Keywords: Ion-transport mechanisms, High entropy alloy, Passive film, ToF-SIMS

\section{Introduction}

Over the past 15 years, a new concept based on the mixing of multi-principal elements has opened the way for the development of new complex metallic materials commonly called high entropy alloys (HEA). This approach proposes to study the central parts of phase diagrams to produce concentrated solid solutions by maximizing the configurational mixing entropy of the system [1,2], which offers a multitude of new alloys to explore [3].

The face centered cubic high entropy alloys (CoCrFeMnNi), including the famous Cantor alloy [1] and its derivatives [4], have been largely studied and present interesting mechanical properties compared to 
conventional alloys [5]. Currently, a significant focus is being made to understand the corrosion resistance of this new class of materials [6-8] and the characterization of the protective surface oxide layers $[9,10]$. Surface analysis, including x-ray photoelectron spectroscopy (XPS) and time-of-flight secondary ion mass spectrometry (ToF-SIMS), has revealed that the air-formed native oxide film and passive film formed in acid medium on $\mathrm{CoCrFeMnNi}$ surfaces present a bilayer stratified structure with a Fe-, $\mathrm{Cr}$ - and Co-rich outer layer, and a Mn- and $\mathrm{Cr}$-rich inner layer, and that nickel is enriched in the modified alloy region underneath the oxide film [10]. A large amount of $\mathrm{Cr}$ species (oxide and hydroxide) is observed for the native (46 at. \%) and passive (65 at. \%) layers, with stronger enrichment of oxidized $\mathrm{Cr}(\mathrm{III})$ species for passive layers.

The high temperature oxidation behaviour of the $\mathrm{CoCrFeMnNi} \mathrm{HEA}$ was studied and found to be largely governed by the oxidation of the $\mathrm{Cr}$ and $\mathrm{Mn}$ elements [11]. $\mathrm{Cr}_{2} \mathrm{O}_{3}$ and $\mathrm{Mn}_{2} \mathrm{O}_{3}$ were the main oxides formed at $900^{\circ} \mathrm{C}$, while $(\mathrm{Mn}, \mathrm{Cr})_{3} \mathrm{O}_{4}$ and $\mathrm{Mn}_{3} \mathrm{O}_{4}$ were the main oxides formed at $1000^{\circ} \mathrm{C}$ and $1100^{\circ} \mathrm{C}$, respectively. Kai et al. [12] studied the effect of oxygen-containing atmospheres on oxidation at $950^{\circ} \mathrm{C}$ and found that the oxidation kinetics followed a parabolic rate law, with the oxidation rate constants increasing with increasing oxygen partial pressure. Oxidation was also investigated at medium temperatures $\left(600-900^{\circ} \mathrm{C}\right)$ in laboratory air [13]. At $600^{\circ} \mathrm{C}$, the main grown oxide was $\mathrm{Mn}_{2} \mathrm{O}_{3}$, however with a thin inner layer of $\mathrm{Cr}_{2} \mathrm{O}_{3}$. $\mathrm{Mn}_{2} \mathrm{O}_{3}$ persists up to $800^{\circ} \mathrm{C}$ but transforms to $\mathrm{Mn}_{3} \mathrm{O}_{4}$ at $900^{\circ} \mathrm{C}$. The oxidation rate was initially linear but became parabolic at longer oxidation time. Lower temperature $\left(120^{\circ} \mathrm{C}\right.$ and $300^{\circ} \mathrm{C}$ ) oxidation of the $\mathrm{Ni}_{38} \mathrm{Cr}_{22} \mathrm{Fe}_{20} \mathrm{Mn}_{10} \mathrm{Co}_{10}$ (at.\%) HEA showed that the oxide film consists of a Cr-rich inner oxide and a Fe, Mn, Co and Ni-rich outer oxide, and the enrichment of Ni and depletion of $\mathrm{Cr}$ in the oxide/metal interface was observed at $300^{\circ} \mathrm{C}$ [14].

Although several studies have investigated oxide film composition and the associated oxidation kinetics at different temperatures [6, 11-19], the ion-transport process that governs oxide growth on such complex alloys is rarely elucidated. ToF-SIMS analysis can make use of the isotopic tracer ${ }^{18} \mathrm{O}$ in order to identify the predominant ion transport during surface oxidation, by exposing the sample to a 'labelled' ${ }^{18} \mathrm{O}_{2}$ atmosphere and depth profiling the oxidized surface. Using this analytical approach, Poulain et al. [20] investigated ion transport for $\mathrm{Cr}_{2} \mathrm{O}_{3}$ growing on a pure $\mathrm{Cr}$ substrate at $300{ }^{\circ} \mathrm{C}$ and under low oxygen pressure. In addition, the ion transport mechanisms in oxide films pre-formed in varying conditions can also be studied using ToF-SIMS depth profiling labelled with ${ }^{18} \mathrm{O}$ isotope. On Ni-based alloy, the results 
revealed that inward anion diffusion governs the oxide growth on the surface pre-oxidized in high temperature water [21]. On 304L and 316L stainless steels, it was shown that cation diffusion is the main mechanism responsible for oxide growth on surfaces pre-passivated by anodic polarization in aqueous acid solution, and that on $316 \mathrm{~L}$ the oxidation rate is markedly slower than on a surface precovered by an air-formed native oxide film [22, 23].

CoCrFeMnNi HEA is a novel alloy compared to traditional stainless steels, and it could be an alternative to replace stainless steels in industrial applications due to its excellent mechanical properties $[24,25]$. Several studies have investigated its corrosion behaviour and compared it with that of most common stainless steels $[10,26,27]$. However, none of them has addressed the oxide film thermal stability or the transport mechanisms governing the oxide growth. The understanding of the corrosion resistance properties and oxide growth mechanisms of the $\mathrm{CoCrFeMnNi}$ alloy is thus of primary importance for a comparison with stainless steels (304L and 316L).

In the present work, this analytical approach was applied to provide new insight into the ion transport mechanism in the passive oxide film formed on the CoCrFeMnNi HEA. The passive oxide film was pre-formed in sulfuric acid aqueous solution by electrochemical anodic polarization in the passive range, and was further oxidised (a procedure denoted re-oxidation in this paper) at elevated temperature in low oxygen $\left({ }^{18} \mathrm{O}_{2}\right)$ pressure and analysed in situ by ToF-SIMS depth profiling. The only driving force during in-situ re-oxidation experiment is the chemical gradient since no electric field is applied. Thus, this experiment does not provide data on the kinetics of a passive oxide film growth, but it is perfectly suitable to reveal the type of defects (anionic and/or cationic) present in the passive film and governing the passive oxide film growth. In addition, the thermal stability of the passive film in high vacuum was studied from room temperature (RT) to $200^{\circ} \mathrm{C}$, in order to determine an appropriate temperature for the re-oxidation experiments.

\section{Experimental}

\section{Sample preparation}

The single fcc phase equimolar $\mathrm{CoCrFeMnNi}$ HEA was prepared from pure $\mathrm{Fe}, \mathrm{Co}, \mathrm{Cr}$, $\mathrm{Ni}$ and $\mathrm{Mn}$ ingots (purity exceeding $99.9 \mathrm{wt} \%$ ) by high frequency electromagnetic induction melting in a watercooled copper crucible under He atmosphere. The high frequency induction melting was carried out 
in a $50 \mathrm{~kW}$ Fives Celes (France) furnace operating at a frequency of $150 \mathrm{kHz}$. Detailed manufacturing and heat treatment process have been described previously [10, 28]. Finally, after mechanical and thermal treatment, a grain size of about $30 \mu \mathrm{m}$ has been obtained. The sample surface was mechanically polished down to $0.25 \mu \mathrm{m}$ with diamond paste and then successively washed with acetone, ethanol and water in ultrasonic bath for 10 mins. The sample was then dried in compressed air, and used without any aging in air for electrochemical passivation.

A Gamry electrochemical workstation was used for passivation in a standard three-electrode cell with an Au counter-electrode and a saturated calomel electrode as reference electrode. The electrolyte was a $0.05 \mathrm{M} \mathrm{H}_{2} \mathrm{SO}_{4}$ aqueous solution prepared with ultrapure chemicals (VWR) and ultra pure water (Merk Millipore). Before treatment, the solution was deaerated by Ar bubbling for 30 minutes. The native oxide-covered sample was left at open circuit potential for $30 \mathrm{~min}$ before stepping the potential at 0.4 V/SCE. This passivation potential corresponds to the middle of the passive region in which the passive current is minimum as observed from polarization curves [10]. After $1 \mathrm{~h}$ of anodic passivation, applied potential was cut-off and the sample was emerged, rinsed with ultra-pure water and dried with compressed air. Sample was then transferred to ultra-high vacuum (UHV) for surface analysis after aging for 18 hours in air.

\section{ToF-SIMS investigation}

ToF-SIMS depth profiles were obtained using a ToF-SIMS 5 spectrometer (IONTOF GmbH - Münster, Germany) with a base pressure of $10^{-9} \mathrm{mbar}$. A pulsed $25 \mathrm{keV} \mathrm{Bi}^{+}$primary ion source was employed for static analysis, delivering $1.2 \mathrm{pA}$ of target current over a $100 \times 100 \mu \mathrm{m}^{2}$ area. The pulsed primary ion beam was used in the bunched mode, with pulse width of $1.2 \mathrm{~ns}$. Depth profiling was carried out by interlacing static analysis with sputtering using a $0.5 \mathrm{keV} \mathrm{Cs}^{+}$sputter beam giving a $17 \mathrm{nA}$ target current over a $300 \times 300 \mu \mathrm{m}^{2}$ area. $\mathrm{A} \mathrm{Cs}^{+}$ion beam was selected for sputtering due to its low contribution to the secondary ionization yield. Both $\mathrm{Bi}^{+}$and $\mathrm{Cs}^{+}$ion beams were impinged on the sample surface at an angle of $45^{\circ}$ and were aligned in such a way that the analyzed ions were taken from the center of the sputtered crater in order to avoid edge effects. 


\begin{tabular}{|c|c|c|}
\hline & Species & Characteristic ion \\
\hline \multirow{4}{*}{$\begin{array}{l}\text { Metallic } \\
\text { substrate }\end{array}$} & Metallic Mn & ${ }^{55} \mathrm{Mn}_{2}^{-}$ \\
\hline & Metallic Fe & ${ }^{56} \mathrm{Fe}_{2}^{-}$ \\
\hline & Metallic Ni & ${ }^{58} \mathrm{Ni}_{2}^{-}$ \\
\hline & Metallic Co & ${ }^{59} \mathrm{Co}_{2}^{-}$ \\
\hline \multirow{4}{*}{$\begin{array}{l}\text { Pre-formed oxide } \\
\qquad\left({ }^{16} \mathrm{O}\right)\end{array}$} & Chromium oxide & ${ }^{52} \mathrm{Cr}^{16} \mathrm{O}_{2}^{-}$ \\
\hline & Manganese oxide & ${ }^{55} \mathrm{Mn}^{16} \mathrm{O}_{2}^{-}$ \\
\hline & Iron oxide & ${ }^{56} \mathrm{Fe}^{16} \mathrm{O}_{2}^{-} ;{ }^{56} \mathrm{Fe}_{2}{ }^{16} \mathrm{O}_{3}-$ \\
\hline & Cobalt oxide & ${ }^{59} \mathrm{Co}^{16} \mathrm{O}_{2}^{-}$ \\
\hline \multirow{4}{*}{$\begin{array}{l}\text { Newly-formed oxide } \\
\qquad\left({ }^{18} \mathrm{O}\right)\end{array}$} & Chromium oxide & ${ }^{52} \mathrm{Cr}^{18} \mathrm{O}_{3}^{-}$ \\
\hline & Manganese oxide & ${ }^{55} \mathrm{Mn}^{18} \mathrm{O}_{3}^{-}$ \\
\hline & Iron oxide & ${ }^{56} \mathrm{Fe}^{18} \mathrm{O}_{2}^{-}$ \\
\hline & Cobalt oxide & ${ }^{59} \mathrm{Co}^{18} \mathrm{O}_{2}^{-}$ \\
\hline
\end{tabular}

Table 1 ToF-SIMS characteristic ions used to characterize the composition and stratification of the oxide film formed on the CoCrFeMnNi HEA

ToF-SIMS depth profiles were recorded to determine the composition and layer structure of the surface oxide film. The characteristic ions were selected as shown in Table 1. The pre-formed iron oxide is associated to two different characteristic ions $\left(\mathrm{Fe}^{16} \mathrm{O}_{2}{ }^{-}\right.$with high intensity and $\mathrm{Fe}_{2}{ }^{16} \mathrm{O}_{3}{ }^{-}$with low intensity). Despite the high mass resolution $(\mathrm{M} / \mathrm{dM}=7500)$ that allows us to locate the maximum intensity of each single peak, the characteristic ion of newly formed chromium oxide $\left(\mathrm{Cr}^{18} \mathrm{O}_{2}^{2}, 87.93\right.$ amu) slightly overlaps in the mass spectrum with the tail of characteristic ion of the pre-formed iron oxide $\left(\mathrm{Fe}^{16} \mathrm{O}_{2}{ }^{-}, 87.92 \mathrm{amu}\right)$. To avoid this problem, $\mathrm{Cr}^{18} \mathrm{O}_{3}{ }^{-}(105.93 \mathrm{amu})$ and $\mathrm{Fe}_{2}{ }^{16} \mathrm{O}_{3}{ }^{-}(159.85 \mathrm{amu})$ ions (that do not suffer from overlapping) were used to characterize the oxide growth during reoxidation in ${ }^{18} \mathrm{O}_{2}$ of the oxide pre-formed in $\mathrm{H}_{2} \mathrm{SO}_{4}$. It should be noted that the selected ions do not reflect the real stoichiometry of the species constituting the sample but are the appropriate markers of the studied species. Since ToF-SIMS is a non-quantitative technique (due to strong matrix effect on secondary ion emission), the intensities of the plotted ions in the depth profiles cannot be compared directly and do not reflect the concentrations of the associated species in the sample. However, the intensity variations of a single signal reflect mainly the in-depth variations of the concentration. The depth profiles are plotted versus sputtering time. The sputtering rate has been calculated knowing for the passivated $\mathrm{CoCrFeMnNi}$ sample: (i) the total oxide layer thickness previously measured from XPS [10], and (ii) the position of the metal/oxide interface determined from each depth profile analysis by 
using the maximum intensity of the $\mathrm{Ni}_{2}{ }^{-}$signal. Assuming a constant sputtering rate $(0.015 \mathrm{~nm} / \mathrm{s})[22,23]$ in the oxide, independent of the oxide layer composition, the sputtering time directly translates into oxide thickness.

The passivated sample was first analysed immediately after introduction into the ToF-SIMS analysis chamber, and prior to the application of heat treatment. The specimen was then heated up to a temperature of $170 \pm 1^{\circ} \mathrm{C}$ (an IONTOF heating stage was used). A precision leak valve was then used to introduce a constant and low ${ }^{18} \mathrm{O}_{2}$ pressure into the analysis chamber, such that the partial pressure $\mathrm{p}\left({ }^{18} \mathrm{O}_{2}\right)$ was constant at $1 \times 10^{-5}$ mbar. After the designated oxidation time had elapsed, the leak valve was closed while the sample temperature was maintained at $170 \pm 1{ }^{\circ} \mathrm{C}$, and the chamber immediately pumped down to the base pressure $\left(10^{-9} \mathrm{mbar}\right)$, in order to record the ToF-SIMS depth profiles.After depth profiles analysis, oxygen was then introduced at the same partial pressure for further oxidation and the analytical protocol repeated for each subsequent oxidation step. Due to the destructive nature of sputtering, each depth profile was collected at a different area of the sample surface, unperturbed by prior analysis.

\section{Results and discussion}

\section{Bilayer structure and thermal stability of the passive oxide film}

In order to investigate the ion transport process, an appropriate temperature must be chosen. Indeed, if the temperature is too low, ion transport is slow and a long time is required to observe oxide film growth, while if the temperature is too high, the composition and structure of the oxide film are not stable and change too rapidly. Thus, we need to determine the thermal stability of the passive film at different temperatures in the UHV environment.

The passivated CoCrFeMnNi HEA sample was introduced in the ToF-SIMS analysis chamber under a base pressure lower than $10^{-8}$ mbar. The experiments were performed through stepwise heating from $50^{\circ} \mathrm{C}$ to $200^{\circ} \mathrm{C}$ with steps of $50^{\circ} \mathrm{C}$. The time used for increasing the temperature $\left(50^{\circ} \mathrm{C}\right.$ step $)$ is less than $1 \mathrm{~min}$. At each temperature step, ToF-SIMS depth profiles were acquired after a heating time of 30 minutes. Each analysis was obtained on a new area of the sample surface. ToF-SIMS is a surface and thin film analytical technique that does not provide direct quantitative determination of the concentration of each species. However, the in-depth trends in the intensities of the different oxides' 
signals allow us to discuss the evolution with thickness of the amount of the different species constituting the oxide scale when the matrix remains the same. In addition, our previous research [10], reporting XPS data, gave the quantitative characterization of the composition of the CoCrFeMnNi HEA surface passivated in the same conditions of anodic polarization in sulfuric acid. Coupling between XPS and ToF-SIMS was shown to be a very powerful methodology to quantitatively describe the oxide scale formed on the CoCrFeMnNi HEA surface.

(a)

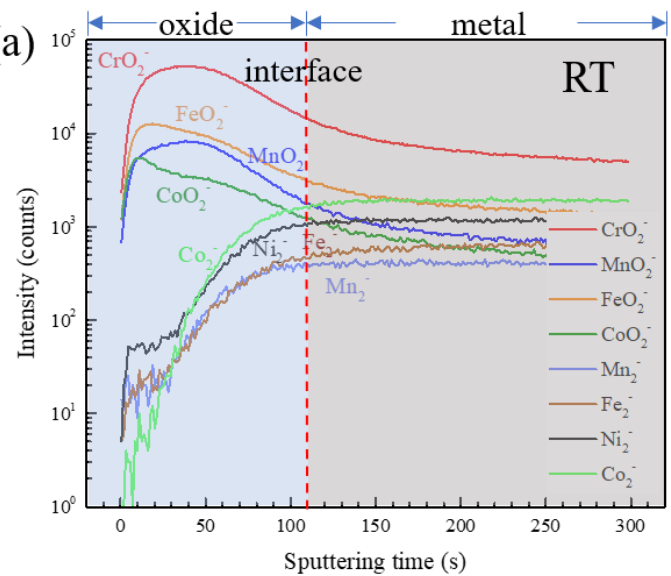

(c)

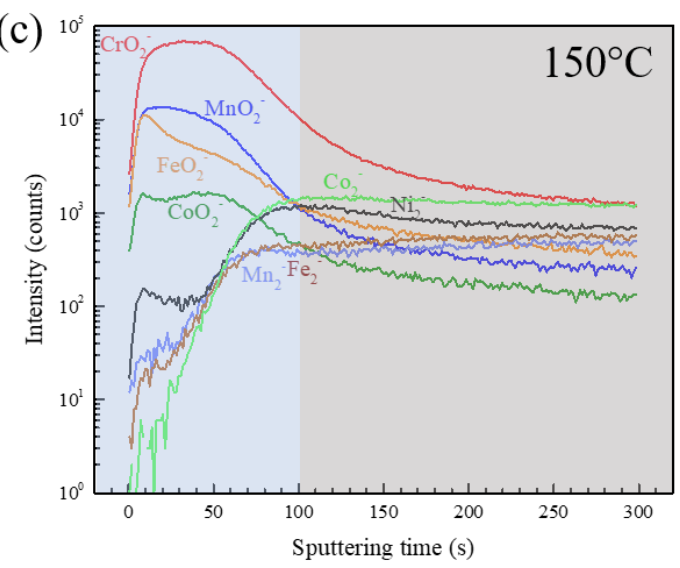

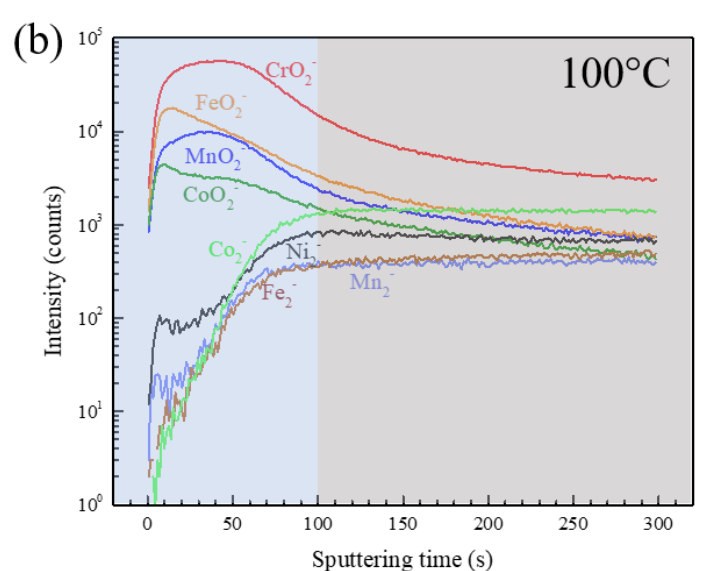

(d)

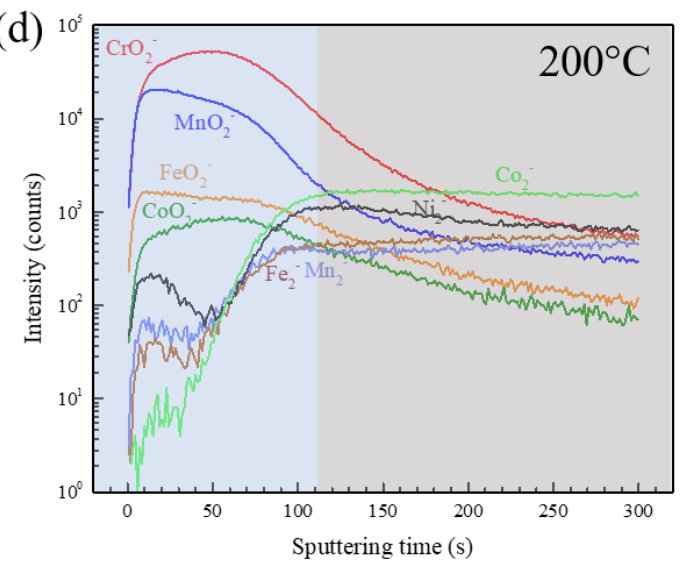

Fig.1 ToF-SIMS depth profiles of the passive film pre-formed on CoCrFeMnNi HEA surfaces in sulfuric acid solution and recorded at different heating temperatures in ultra-high vacuum: (a) RT; (b) $100^{\circ} \mathrm{C}$; (c) $150^{\circ} \mathrm{C}$ and (d) $200^{\circ} \mathrm{C}$

Fig. 1 shows the ToF-SIMS negative ion depth profiles of the passivated surface recorded at RT, $100^{\circ} \mathrm{C}$, $150^{\circ} \mathrm{C}$ and $200^{\circ} \mathrm{C}$. The depth profiles at $50^{\circ} \mathrm{C}$ are not shown since they were identical to those obtained at RT. In each graph, the $\mathrm{Ni}_{2}{ }^{-}$ion profile is used to define the interface between oxide film and metallic substrate. Our previous XPS results on this CoCrFeMnNi HEA [10] and stainless steels [29] showed that a Ni-enriched modified layer exists in the alloy beneath the surface oxide. This metallic Ni 
enrichment is characterized by a hump in the $\mathrm{Ni}_{2}{ }^{-} \mathrm{ToF}-\mathrm{SIMS}$ signal close to the metal/oxide interface. Thus, the maximum intensity of the $\mathrm{Ni}_{2}{ }^{-}$signal is used to locate the metal/oxide interface in the present study, like previously on stainless alloys [21-23]. The $\mathrm{NiO}_{2}{ }^{-}$ion profiles are not plotted in Fig.1, since their intensities are extremely low compared to those of the $\mathrm{CrO}_{2}{ }^{-}, \mathrm{FeO}_{2}^{-}, \mathrm{CoO}_{2}{ }^{-}$and $\mathrm{MnO}_{2}{ }^{-}$ion profiles. It means that nickel oxide is present only at trace level in the oxide film.

At RT, in Fig.1(a), the interface between oxide film and metallic substrate is defined at $110 \mathrm{~s}$ of sputtering time. In the oxide film region, the $\mathrm{FeO}_{2}{ }^{-}$profile peaks in the outer part of the oxide region and decreases slowly through the inner part with ongoing sputtering. Oxidized iron is therefore mainly located in the outer part of the oxide film. The presence of Fe oxide in small amount in the inner part of the oxide cannot be excluded. The $\mathrm{CoO}_{2}^{-}$profile also has its maximum in the outer oxide region, indicating that oxidized cobalt is also mainly located in the outer part of the oxide film. For the $\mathrm{CrO}_{2}^{-}$ profile, it exhibits a wide peak throughout the oxide region, with a maximum located in the inner part. Oxidized chromium is thus mainly distributed in the inner part of the oxide film. A small amount in the outer part cannot be excluded. Finally, the $\mathrm{MnO}_{2}{ }^{-}$profile has its maximum in the inner part of the oxide region, indicating that manganese oxide is preferentially located in the inner part of the oxide film, like chromium oxide. As already observed [10] and based on this ToF-SIMS depth profile analysis, it is thus confirmed that the passive oxide film is stratified and can be described by a bilayer oxide structure mainly composed of $\mathrm{Fe}$ and $\mathrm{Co}$ oxides in the outer layer and $\mathrm{Cr}$ and $\mathrm{Mn}$ oxides in the inner layer. Upon heating at $100^{\circ} \mathrm{C}$ in vacuum (Fig.1(b)), the depth profiles of the $\mathrm{CrO}_{2}^{-}, \mathrm{FeO}_{2}^{-}, \mathrm{CoO}_{2}^{-}$and $\mathrm{MnO}_{2}{ }^{-}$ions do not markedly change, meaning that the bilayer structure of the oxide film remains stable at $100^{\circ} \mathrm{C}$.

At $150^{\circ} \mathrm{C}$, as shown in Fig.1(c), the $\mathrm{FeO}_{2}^{-}$profile still exhibits a peak in the outer part of the oxide region. However, the intensity in the inner part is reduced compared to that in the depth profiles at RT and $100^{\circ} \mathrm{C}$, indicating that $\mathrm{Fe}$ oxide is more concentrated in the outer part of the oxide film. The $\mathrm{CoO}_{2}{ }^{-}$ depth profile, although still showing a small peak in the outer part of the oxide region, is greatly reduced in intensity compared to the profiles at RT and $100^{\circ} \mathrm{C}$, showing that little Co oxide is left in the film after heating up to $150^{\circ} \mathrm{C}$. The peak of the $\mathrm{MnO}_{2}^{-}$profile has shifted to the outer part of the oxide region and the intensity decreases slowly through the inner oxide region, indicating that manganese oxide is now mainly located in the outer part of the oxide film with small amount left in the inner part. The respective decrease and increase of Fe oxide and Mn oxide suggest the reduction of less stable Fe oxide species with some oxygen consumed by transformation of Mn oxide to a more stable oxide $[30,31]$. 
This would be accompanied by redistribution of the Mn oxide species between the inner and outer parts of the film. The $\mathrm{CrO}_{2}^{-}$profile shows no marked change, meaning that $\mathrm{Cr}$ oxide is not reduced and remains mainly distributed in the inner region of the film.

At $200^{\circ} \mathrm{C}$ (Fig.1(d)), the $\mathrm{FeO}_{2}{ }^{-}$profile is markedly reduced in intensity still with its maximum in the outer oxide region, showing that Fe oxide is increasingly reduced with smaller amounts left in the oxide film. Meanwhile, the $\mathrm{MnO}_{2}^{-}$profile increases in intensity both in the outer and inner oxide regions, confirming the exchange reaction between iron oxide and manganese oxide with more Mn oxide located in the outer part of the film. The $\mathrm{CoO}_{2}^{-}$profile shows further decrease in intensity, indicative of trace level of Co oxide in the film. The $\mathrm{CrO}_{2}^{-}$profile is slightly decreased in intensity in the outer part of the film, showing that its reduction is initiated at the outer surface at the interface with the UHV environment.

Based on this depth profile analysis performed at different heating temperatures, we can say that the passive film is thermally stable under UHV below $100^{\circ} \mathrm{C}$ with a bilayer structure mainly constituted of $\mathrm{Fe}$ and Co oxides in the outer layer and $\mathrm{Mn}$ and $\mathrm{Cr}$ oxides in the inner layer. At $150^{\circ} \mathrm{C}$, the passive film is slightly altered with more Mn oxide in the outer part and Fe oxide reduced mostly in the inner part of the film. Co oxide subsists in small amounts. At $200^{\circ} \mathrm{C}$, the bilayer structure of the passive film persists but the composition is greatly changed. Mn oxide and Cr oxide are the main constituents of the outer and inner layers, respectively. Only traces of Fe and Co oxides remain in the film.

Thus, a temperature around $150^{\circ} \mathrm{C}$ would be a good choice to carry out the re-oxidation experiments, since the composition of the film shows only small alteration after annealing for $30 \mathrm{~min}$. After a series of re-oxidation tests performed at different temperatures around $150^{\circ} \mathrm{C}$, the temperature of $170^{\circ} \mathrm{C}$ was selected to perform the re-oxidation experiments. This temperature is a good compromise to ensure sufficiently fast kinetics of ion transport and stability of the pre-formed passive film, allowing us to investigate ion transport in a quasi-intact passive film.

The structure of the oxide scale formed on $\mathrm{CoCrFeMnNi}$ is unmodified up to a temperature around $170^{\circ} \mathrm{C}$, which is much less than the thermal stability of the passive films formed on $316 \mathrm{~L}$ stainless steel (SS) [32] that shows stable structure up to $300^{\circ} \mathrm{C}$. This could be assigned to the composition of the alloy itself. The structure modification on $\mathrm{CoCrFeMnNi}$ alloy at $170^{\circ} \mathrm{C}$ is assigned to the growth of $\mathrm{Mn}$ oxide at the expense of the $\mathrm{Fe}$ oxide at $170^{\circ} \mathrm{C}$. The absence of $\mathrm{Mn}$ in the SS substrate makes its oxide scale more thermally stable than the one formed on CoCrFeMnNi alloy. 


\section{Ion transport mechanisms}

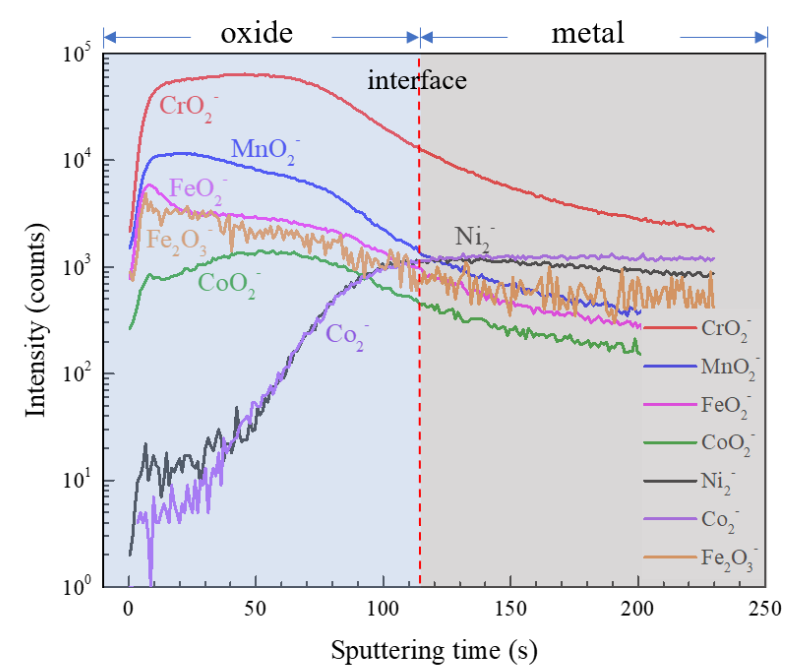

Fig.2 ToF-SIMS depth profiles of the passive film pre-formed on CoCrFeMnNi HEA surfaces in sulfuric acid solution and recorded after heating up directly from $\mathrm{RT}$ to $170^{\circ} \mathrm{C}$ in ultra-high vacuum (The intensity of $\mathrm{Fe}_{2}{ }^{16} \mathrm{O}_{3}^{-}$signal is multiplied by 50 )

Fig. 2 shows the characteristic ToF-SIMS depth profiles for the electrochemically pre-passivated surface film after increasing the temperature to $170^{\circ} \mathrm{C}$ under UHV. The passive film thickness is estimated to be $1.7 \mathrm{~nm}$ from XPS data [10]. The structure of the passive film remains bilayered as indicated by the $\mathrm{FeO}_{2}{ }^{-}$and $\mathrm{MnO}_{2}{ }^{-}$signals that reach their maximum intensities in the outer part of the oxide region whereas the $\mathrm{CrO}_{2}^{-}$and $\mathrm{CoO}_{2}^{-}$signal have their maximum in the inner part. However, some compositional changes, including the lower intensity of the $\mathrm{FeO}_{2}^{-}$ions and the slightly higher intensity of the $\mathrm{MnO}_{2}^{-}$signal in the outer part of the oxide film region are confirmed when increasing the temperature from room temperature to $170^{\circ} \mathrm{C}$. Nevertheless, the structure of the oxide is still a bilayer and the main species in the film are still $\mathrm{Fe}, \mathrm{Cr}$ and Mn oxides. In the following, Co oxide will not be discussed since it is reduced to trace level in the film after heating up to $170^{\circ} \mathrm{C}$. In addition, since the $\mathrm{Fe}_{2} \mathrm{O}_{3}{ }^{-}$depth profile shows the same trend as the $\mathrm{FeO}_{2}{ }^{-}$depth profile, it will be used in the analysis of the re-oxidation experiment because of peak overlapping in the mass spectra between $\mathrm{FeO}_{2}{ }^{-}$and $\mathrm{Cr}^{18} \mathrm{O}_{2}{ }^{-}$ ions. 

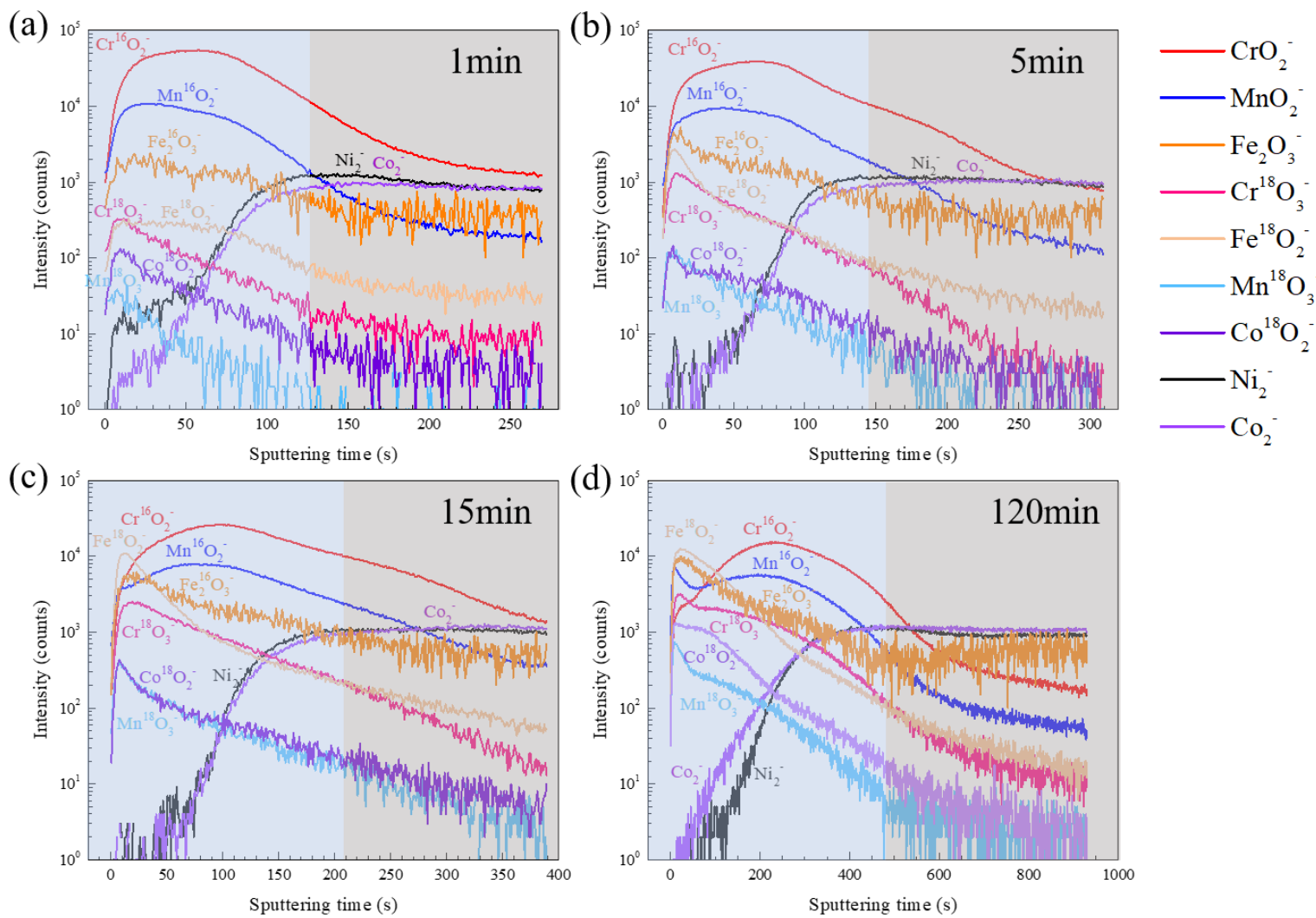

Fig.3 ToF-SIMS depth profiles obtained on the passive film pre-formed on CoCrFeMnNi HEA surfaces in sulfuric acid solution and recorded after re-oxidation at $170{ }^{\circ} \mathrm{C}$ by exposure to $18 \mathrm{O} 2$ at low pressure for (a) $1 \mathrm{~min}$, (b) $5 \mathrm{~min}$, (c) $15 \mathrm{~min}$ and (d) $2 \mathrm{~h}$ (The intensity of $\mathrm{Fe}_{2}{ }^{16} \mathrm{O}_{3}{ }^{-}$signal is multiplied by 50 )

Fig. 3 shows the ToF-SIMS depth profiles obtained on the pre-passivated CoCrFeMnNi HEA surface after exposure to isotopic ${ }^{18} \mathrm{O}_{2}$ gas at low pressure $\left(10^{-5} \mathrm{mbar}\right)$ and $170^{\circ} \mathrm{C}$ for increasing times. After 1 min of re-oxidation (Fig.3(a)), the metal/oxide interface, which is defined by the maximum intensity of the $\mathrm{Ni}_{2}^{-}$signal, is located at $125 \mathrm{~s}$ of sputtering time, which corresponds to an equivalent thickness of $1.9 \mathrm{~nm}$. By examining the signals of the newly formed oxides, including the $\mathrm{Cr}^{18} \mathrm{O}_{3}{ }^{-}, \mathrm{Fe}^{18} \mathrm{O}_{2}{ }^{-}, \mathrm{Mn}^{18} \mathrm{O}_{3}{ }^{-}$ and $\mathrm{Co}^{18} \mathrm{O}_{2}-$ ions, one can locate the newly formed oxides (grown by re-oxidation in ${ }^{18} \mathrm{O}_{2}$ gas) at the surface of the pre-formed passive film. Thus, the main ion transport mechanism for the passive film pre-formed at RT in sulfuric acid solution on CoCrFeMnNi HEA surfaces is outward cation diffusion.

After a re-oxidation time of 5 min (Fig.3(b)), the oxide film is thicker, $2.2 \mathrm{~nm}$ as obtained from the oxide/metal interface positioned at $145 \mathrm{~s}$ of sputtering time. The intensities of the ions characteristic of the newly formed $\mathrm{Cr}, \mathrm{Fe}, \mathrm{Mn}$ and $\mathrm{Co}$ oxides $\left(\mathrm{M}^{18} \mathrm{O}_{2}^{-}\right)$increase with their maximum still at the outer surface, thus confirming that the main ion transport mechanism governing oxide growth is the diffusion 
of cations through the pre-formed passive film to the outer oxide surface. With in-depth profiling, these intensities decrease slowly, with however a small shoulder at around $100 \mathrm{~s}$ of sputtering time, i.e. in the inner oxide region. The presence of shoulders indicates that there are smaller amounts of the newly formed oxides $\left(\mathrm{M}^{18} \mathrm{O}_{2}^{-}\right)$that also form close to the oxide/metal interface. This is indicative of slower inward oxygen diffusion causing the minor growth of newly formed oxides $\left(\mathrm{M}^{18} \mathrm{O}_{2}^{-}\right)$in the inner oxide region near the oxide/metal interfacial region. The presence of pathways for slower anionic transport in the pre-formed passive film enables oxygen to diffuse inward and to react with outward diffusing cations. Focusing on the pre-formed oxide signals $\left(\mathrm{Fe}_{2}{ }^{16} \mathrm{O}_{3}{ }^{-}, \mathrm{Cr}^{16} \mathrm{O}_{2}^{-}\right.$, and $\left.\mathrm{Mn}^{16} \mathrm{O}_{2}{ }^{-}\right)$, we can observe a shallow depression in the decreasing profiles around $120 \mathrm{~s}$ of sputtering time, nearly concomitant with the formation of new oxide close to the metal/oxide interface. This suggests that the pre-formed oxide is displaced by the newly formed oxide. The displacement of the pre-formed oxide in the inner part of the film is also consistent with the shoulder that appears around $170 \mathrm{~s}$ time in the $\mathrm{Cr}^{16} \mathrm{O}_{2}{ }^{-}$and $\mathrm{Mn}^{16} \mathrm{O}_{2}{ }^{-}$ profiles. Overall, the pre-formed inner oxide region is broadened as a result of the minority formation of new oxides in the inner part of the passive film by inward oxygen diffusion.

After 15 min of re-oxidation (Fig.3(c)), the $\mathrm{Ni}_{2}{ }^{-}$signal enables to position the metal/oxide interface at $210 \mathrm{~s}$ of sputtering time, corresponding to a total oxide layer thickness of $3.2 \mathrm{~nm}$. The oxide film is clearly growing. Looking at the depth profiles of newly formed oxide signals, their maximum intensities remain mainly located at the outer surface, and the intensities, especially for the $\mathrm{Cr}^{18} \mathrm{O}_{3}{ }^{-}$depth profile, slowly decreases through the pre-formed passive oxide film. This confirms that the newly formed oxides $\left({ }^{18} \mathrm{O}\right)$ are still growing mainly at the oxide/gas interface due to faster outward cation diffusion through the oxide. The formation of new oxides within the oxide scale close to the metal/oxide interface (as observed and discussed above for 5 min reoxidation) is still ongoing due to the slower inward anion diffusion via anionic pathways. However, the formation of the new oxides builds a ${ }^{16} \mathrm{O}$ and ${ }^{18} \mathrm{O}$ concentration gradient, which is then balanced by the oxygen isotopic exchange taking place in the growing oxide scale.

After 120 min of re-oxidation (Fig.3(d)), the oxide thickness continues to increase and reaches $7 \mathrm{~nm}$, as determined from the oxide/metal positioned at $\sim 450 \mathrm{~s}$ of sputtering time. The newly formed oxides still peak in the outer surface, due to faster outward cation diffusion. Within the oxide scale, the depth profiles of newly formed and pre-formed oxides are becoming similar in shape and intensity, which we 
interpret as mainly due to isotopic exchange increasingly balancing the concentration gradient between the newly formed ${ }^{18} \mathrm{O}$ and pre-formed ${ }^{16} \mathrm{O}$ oxides.

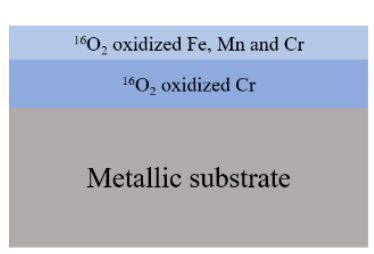

Before re-oxidation

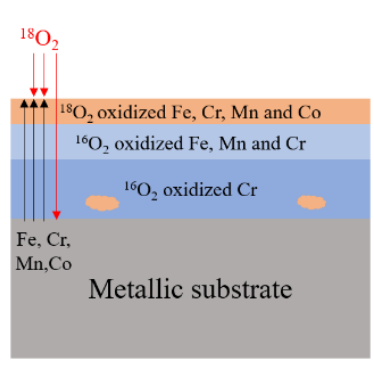

$1 \mathrm{~min}$ re-oxidation

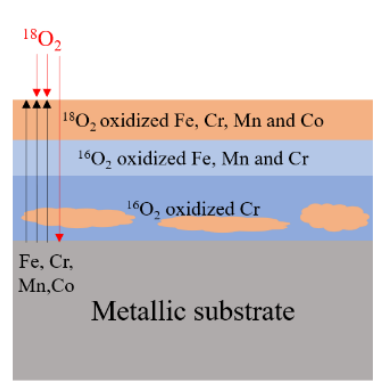

5 min re-oxidation

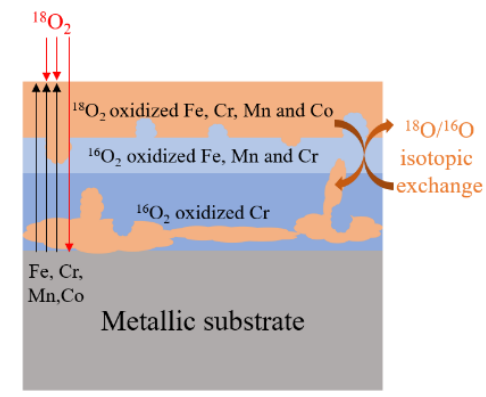

Longer than $5 \mathrm{~min}$ re-oxidation

Fig.4 Scheme of the ion transport mechanisms for re-oxidation at $170{ }^{\circ} \mathrm{C}$ in low pressure ${ }^{18} \mathrm{O}_{2}$ gas of $\mathrm{CoCrFeMnNi} \mathrm{HEA}$ surfaces prepassivated in sulfuric acid solution

Fig.4 presents a scheme of the ion transport mechanisms for the passive film pre-formed on the CoCrFeMnNi HEA as deduced from ToF-SIMS depth profiling analysis performed at $170^{\circ} \mathrm{C}$ in low pressure ${ }^{18} \mathrm{O}_{2}$ gas. As demonstrated above, the main mechanism governing oxide growth upon reoxidation of the pre-passivated surface is outward cation diffusion, meaning that $\mathrm{Fe}, \mathrm{Cr}, \mathrm{Mn}$ and $\mathrm{Co}$ cations diffuse from the metallic substrate to the oxide/gas interface and react with oxygen $\left({ }^{18} \mathrm{O}_{2}\right)$. Thus, the newly formed oxides are predominantly located at the outer oxide/gas interface. In addition, inward anion diffusion also takes place as minority ionic transport process and small amount of newly formed oxides grow in the inner part of the passive film near the oxide/metal interfacial region. With increasing re-oxidation time, oxide growth still proceeds dominantly at the oxide/gas interface owing to faster outward cationic transport through the pre-formed passive film. The ${ }^{18} \mathrm{O} /{ }^{16} \mathrm{O}$ concentration gradients generated between new oxide formation at the external surface and near the oxide/metal interface and the pre-formed passive film regions are balanced by oxygen isotopic exchange. 


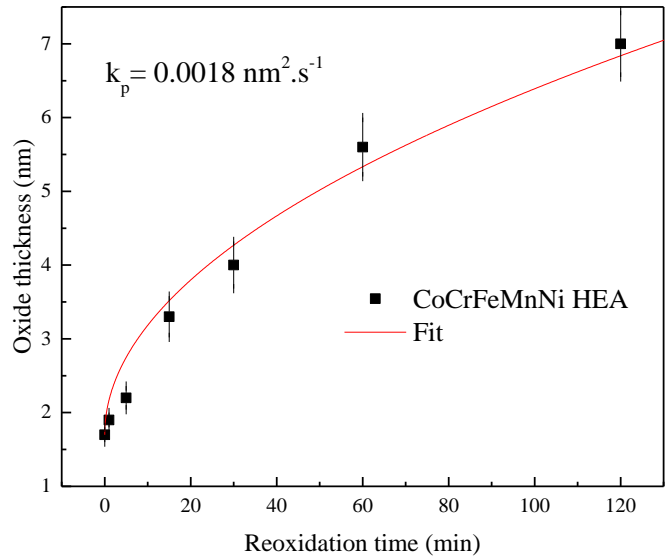

Fig.5 Re-oxidation kinetics (experimental data and fit) for the CoCrFeMnNi HEA surface pre-passivated in sulfuric acid solution

The time evolution of the thickness of the oxide layer, measured by ToF-SIMS during re-oxidation at $170 \mathrm{C}$ under low pressure oxygen gas of the CoCrFeMnNi surface pre-passivated in sulfuric acid solution, is shown in Fig.5. The thickness of the oxide film is defined by the maximum intensity of the $\mathrm{Ni}_{2}{ }^{-}$depth profile. However, due to surface roughness, the metal/oxide interface cannot be viewed as a sharp interface. Thus, it has been considered that the interface region ranges between $100 \%$ and $99 \%$ of the maximum intensity of the $\mathrm{Ni}_{2}{ }^{-}$signal. The error bars reflect the uncertainty of the interface location due to roughness.

The kinetics of re-oxidation is well fitted by a parabolic law described by Eq.(1):

$\left(y-y_{0}\right)^{2}=2 k_{p} t$

where $\mathrm{k}_{\mathrm{p}}$ is the parabolic constant, $y_{0}$ the thickness of pre-formed passive oxide film, $y$ the thickness of the oxide film during re-oxidation and the re-oxidation time. Although the newly formed Cr oxide mainly grows at the outer surface, no Cr oxide volatilization was considered for the fit. This is because the experiment was conducted at $170^{\circ} \mathrm{C}$, which is lower than the volatilization temperature $\left(300^{\circ} \mathrm{C}\right)$ of Cr oxide [20].

The value of $\mathrm{k}_{\mathrm{p}}$ derived from the fit in Fig. 5 is $1.8 \times 10^{-3} \mathrm{~nm}^{2} \cdot \mathrm{s}^{-1}$. This value is very similar to the value $\left(1.9 \times 10^{-3} \mathrm{~nm}^{2} \cdot \mathrm{s}^{-1}\right)$ reported for the Ni-based polycrystalline alloy $\mathrm{Ni}-16 \mathrm{Cr}-8 \mathrm{Fe}$, oxidized in low pressure $\mathrm{O}_{2}$ gas $\left(10^{-6} \mathrm{mbar}\right)$ at $250^{\circ} \mathrm{C}$ [33]. However, since the oxidation temperature of the CoCrFeMnNi HEA 
used here is lower than that of the Ni-based alloy, the oxidation rate of the HEA should be higher. This difference could be due to the presence of $\mathrm{Mn}$ in the alloy. Oxidation studies on CoCrFeMnNi HEA demonstrated that $\mathrm{Mn}$ is detrimental to the oxidation resistance $[11,13,14]$.

\section{Conclusion}

The composition and stratified structure of the passive film formed on CoCrFeMnNi HEA surfaces in acid solution and the thermal stability of the film after heating in vacuum from room temperature to $200^{\circ} \mathrm{C}$ has been investigated by ToF-SIMS depth profile analysis. The passive film formed by anodic polarization in $0.05 \mathrm{M} \mathrm{H}_{2} \mathrm{SO}_{4}$ solution at $0.4 \mathrm{~V} / \mathrm{SCE}$ for $1 \mathrm{~h}$ has a bilayer structure with an $\mathrm{Fe}$ and Co rich outer layer and a $\mathrm{Cr}$ and $\mathrm{Mn}$ rich inner layer. The bilayer structure of the passive film is thermally stable in vacuum, however, above $150^{\circ} \mathrm{C}$, compositional changes, including reduction of $\mathrm{Fe}$ and $\mathrm{Co}$ oxides and formation of Mn oxide, are observed.

The ion transport process in the pre-formed passive film was studied by exposing the passivated surface to ${ }^{18} \mathrm{O}_{2}$ gas at low pressure $\left(10^{-5} \mathrm{mbar}\right)$ and $170^{\circ} \mathrm{C}$ in the ToF-SIMS analysis chamber. Based on in situ ToF-SIMS depth profiling performed at different re-oxidation times, growth of newly formed oxides $\left({ }^{18} \mathrm{O}\right.$ species) at the oxide/gas interface revealed that outward cation diffusion is the main transport mechanism. Inward oxygen diffusion causing oxide growth in the inner part of the pre-formed passive film near the oxide/metal interface was identified as a minority transport mechanism. The ${ }^{16} \mathrm{O} /{ }^{18} \mathrm{O}$ isotopic exchange plays a role in the re-oxidation process to balance the oxygen concentration gradient established within the oxide scale between newly formed and pre-formed oxides. The oxide film growth follows the parabolic law, and the value of the parabolic constant is $0.0018 \mathrm{~nm}^{2} \cdot \mathrm{s}^{-1}$.

\section{Declaration of Competing Interest}

The authors declare that they have no known competing financial interests or personal relationships that could have appeared to influence the work reported in this paper.

\section{Data Availability}

The data that support the findings of this study are available from the corresponding authors upon reasonable request. 


\section{Author Statement}

Luntao Wang: Investigation, Validation, Visualization, Writing - Original Draft

Antoine Seyeux: Supervision

Loïc Perriere: Resources

Dimitri Mercier: Supervision, Writing - Review; Editing

Vincent Maurice: Supervision, Writing - Review; Editing, Funding acquisition

Philippe Marcus: Conceptualization, Supervision, Writing - Review Editing, Funding acquisition, Project management

\section{Acknowledgments}

This project has received funding from the European Research Council (ERC) under the European Union's Horizon 2020 research and innovation program (ERC Advanced Grant No. 741123, Corrosion Initiation Mechanisms at the Nanometric and Atomic Scales: CIMNAS). Région Île-de-France is acknowledged for partial funding of the ToF-SIMS equipment.

\section{References}

[1] B. Cantor, I.T.H. Chang, P. Knight, A.J.B. Vincent, Microstructural development in equiatomic multicomponent alloys, Materials Science and Engineering: A, 375-377 (2004) 213-218.

[2] J.W. Yeh, S.K. Chen, S.J. Lin, J.Y. Gan, T.S. Chin, T.T. Shun, C.H. Tsau, S.Y. Chang, Nanostructured high- entropy alloys with multiple principal elements: novel alloy design concepts and outcomes, Advanced Engineering Materials, 6 (2004) 299-303.

[3] D. Miracle, High entropy alloys as a bold step forward in alloy development, Nature communications, 10 (2019) 1-3.

[4] D.B. Miracle, O.N. Senkov, A critical review of high entropy alloys and related concepts, Acta Materialia, 122 (2017) 448-511.

[5] S. Gorsse, D.B. Miracle, O.N. Senkov, Mapping the world of complex concentrated alloys, Acta Materialia, 135 (2017) 177-187.

[6] X.-X. Yu, M.A. Taylor, J.H. Perepezko, L.D. Marks, Competition between thermodynamics, kinetics and growth mode in the early-stage oxidation of an equimolar $\mathrm{CoCrFeNi}$ alloy, Acta Materialia, 196 (2020) 651-659.

[7] Y. Qiu, M. Gibson, H. Fraser, N. Birbilis, Corrosion characteristics of high entropy alloys, Materials science and technology, 31 (2015) 1235-1243.

[8] Y. Qiu, S. Thomas, M.A. Gibson, H.L. Fraser, N. Birbilis, Corrosion of high entropy alloys, npj Materials degradation, 1 (2017) 1-18.

[9] A.Y. Gerard, J. Han, S.J. McDonnell, K. Ogle, E.J. Kautz, D.K. Schreiber, P. Lu, J.E. Saal, G.S. Frankel, J.R. Scully, Aqueous passivation of multi-principal element alloy Ni38Fe20Cr22Mn10Co10: Unexpected high Cr enrichment within the passive film, Acta Materialia, 198 (2020) 121-133. 
[10] L. Wang, D. Mercier, S. Zanna, A. Seyeux, M. Laurent-Brocq, L. Perrière, I. Guillot, P. Marcus, Study of the surface oxides and corrosion behaviour of an equiatomic CoCrFeMnNi high entropy alloy by XPS and ToF-SIMS, Corrosion Science, (2020) 108507.

[11] Y.-K. Kim, Y.-A. Joo, H.S. Kim, K.-A. Lee, High temperature oxidation behavior of Cr-Mn-FeCo-Ni high entropy alloy, Intermetallics, 98 (2018) 45-53.

[12] W. Kai, C.C. Li, F.P. Cheng, K.P. Chu, R.T. Huang, L.W. Tsay, J.J. Kai, The oxidation behavior of an equimolar $\mathrm{FeCoNiCrMn}$ high-entropy alloy at $950^{\circ} \mathrm{C}$ in various oxygen-containing atmospheres, Corrosion Science, 108 (2016) 209-214.

[13] G. Laplanche, U. Volkert, G. Eggeler, E. George, Oxidation behavior of the CrMnFeCoNi highentropy alloy, Oxidation of Metals, 85 (2016) 629-645.

[14] E.J. Kautz, S.V. Lambeets, D.E. Perea, A.Y. Gerard, J. Han, J.R. Scully, J.E. Saal, D.K. Schreiber, Element redistributions during early stages of oxidation in a Ni38Cr22Fe20Mn10Co10 multi-principal element alloy, Scripta Materialia, 194 (2021) 113609.

[15] G.R. Holcomb, J. Tylczak, C. Carney, Oxidation of CoCrFeMnNi High Entropy Alloys, JOM, 67 (2015) 2326-2339.

[16] D. Huang, J. Lu, Y. Zhuang, C. Tian, Y. Li, The role of $\mathrm{Nb}$ on the high temperature oxidation behavior of CoCrFeMnNbxNi high-entropy alloys, Corrosion Science, 158 (2019) 108088.

[17] M.P. Agustianingrum, F.H. Latief, N. Park, U. Lee, Thermal oxidation characteristics of Fex(CoCrMnNi)100-x medium and high-entropy alloys, Intermetallics, 120 (2020) 106757.

[18] S. Shajahan, A. Kumar, M. Chopkar, A. Basu, Oxidation study of CoCrCuFeNiSix high entropy alloys, Materials Research Express, 7 (2020) 016532.

[19] Y. Wang, M. Zhang, J. Jin, P. Gong, X. Wang, Oxidation behavior of CoCrFeMnNi high entropy alloy after plastic deformation, Corrosion Science, 163 (2020) 108285.

[20] C. Poulain, A. Seyeux, S. Voyshnis, P. Marcus, Volatilization and transport mechanisms during Cr oxidation at $300{ }^{\circ} \mathrm{C}$ studied in situ by ToF-SIMS, Oxidation of Metals, 88 (2017) 423-433.

[21] S. Voyshnis, A. Seyeux, S. Zanna, B. Martin-Cabanas, T. Couvant, P. Marcus, Oxide layer growth on nickel-base alloy surfaces in high temperature water and in $\mathrm{O} 2$ studied by ToF-SIMS with isotopic tracers, Corrosion Science, 145 (2018) 212-219.

[22] L. Wang, A. Seyeux, P. Marcus, Ion Transport Mechanisms in the Oxide Film Formed on 316L Stainless Steel Surfaces Studied by ToF-SIMS with $18 \mathrm{O} 2$ Isotopic Tracer, Journal of The Electrochemical Society, 167 (2020) 101511.

[23] L. Wang, S. Voyshnis, A. Seyeux, P. Marcus, Ion transport mechanisms in the passive film formed on 304L stainless steel studied by ToF-SIMS with $18 \mathrm{O}$ isotopic tracer, Corrosion Science, 173 (2020) 108779.

[24] C.C. Tasan, Y. Deng, K.G. Pradeep, M. Yao, H. Springer, D. Raabe, Composition dependence of phase stability, deformation mechanisms, and mechanical properties of the $\mathrm{CoCrFeMnNi}$ high-entropy alloy system, Jom, 66 (2014) 1993-2001.

[25] F. Otto, A. Dlouhý, C. Somsen, H. Bei, G. Eggeler, E.P. George, The influences of temperature and microstructure on the tensile properties of a CoCrFeMnNi high-entropy alloy, Acta Materialia, 61 (2013) 5743-5755.

[26] H. Luo, Z. Li, A.M. Mingers, D. Raabe, Corrosion behavior of an equiatomic CoCrFeMnNi highentropy alloy compared with 304 stainless steel in sulfuric acid solution, Corrosion Science, 134 (2018) 131-139.

[27] J. Pang, T. Xiong, X. Wei, Z. Zhu, B. Zhang, Y. Zhou, X. Shao, Q. Jin, S. Zheng, X. Ma, Oxide 
MnCr2O4 induced pitting corrosion in high entropy alloy CrMnFeCoNi, Materialia, 6 (2019) 100275.

[28] G. Bracq, M. Laurent-Brocq, L. Perrière, R. Pirès, J.-M. Joubert, I. Guillot, The fcc solid solution stability in the Co-Cr-Fe-Mn-Ni multi-component system, Acta Materialia, 128 (2017) 327-336.

[29] Z. Wang, F. Di-Franco, A. Seyeux, S. Zanna, V. Maurice, P. Marcus, Passivation-induced physicochemical alterations of the native surface oxide film on 316L austenitic stainless steel, Journal of The Electrochemical Society, 166 (2019) C3376-C3388.

[30] K. Zumsande, A. Weddeling, E. Hryha, S. Huth, L. Nyborg, S. Weber, N. Krasokha, W. Theisen, Characterization of the surface of $\mathrm{Fe}-19 \mathrm{Mn}-18 \mathrm{Cr}-\mathrm{C}-\mathrm{N}$ during heat treatment in a high vacuum $-\mathrm{An}$ XPS study, Materials Characterization, 71 (2012) 66-76.

[31] A.B. Kaderi, H. Hartmann, A. Besmehn, Observation of Oxide Development from Room Temperature (RT) to $700{ }^{\circ} \mathrm{C}$ Demonstrated by In Situ XPS of Crofer 22 APU Alloy, Oxidation of Metals, 88 (2017) 459-468.

[32] L. Wang, A. Seyeux, P. Marcus, Thermal stability of the passive film formed on 316L stainless steel surface studied by ToF-SIMS, Corrosion Science, 165 (2019) 108395.

[33] X. Wu, S. Voyshnis, A. Seyeux, Y. Chumlyakov, P. Marcus, ToF-SIMS study of oxide films thermally grown on nickel-base alloys, Corrosion Science, 141 (2018) 175-181. 